N.B: Copia ad uso personale. È vietata la riproduzione (totale o parziale) dell'opera con qualsiasi mezzo effettuata e la sua messa a disposizione di terzi, sia in forma gratuita sia a pagamento.

\title{
Geografie della contestazione: il Concilio, l'Italia, l'America latina
}

\author{
di Francesco Mores
}

\section{Provincializzare la contestazione?}

Da ormai un ventennio, pochi studiosi hanno potuto fare a meno di confrontarsi con un tema che è anche il titolo di un libro. Provincializzare l'Europa di Dipesh Chakrabarty è rimasto per molto tempo nell'arsenale di coloro che ritenevano di disporre finalmente delle armi adatte a sconfiggere l'egemonia esercitata dal pensiero occidentale. Trascurandone spesso le conclusioni («spero che sia chiaro da quanto ho scritto che provincializzare l'Europa non può trasformarsi in un progetto volto a sfuggire al pensiero europeo: finito l'imperialismo in Europa, il pensiero europeo è un dono per tutti. Possiamo discutere della sua provincializzazione con uno spirito anticoloniale di gratitudine» ${ }^{2}$ ), i lettori del libro di Chakrabarty si sono limitati a considerarlo un vessillo del pensiero anticoloniale, dimenticando anche affermazioni poste all'inizio del volume:

l'Europa che intendo provincializzare, o decentrare, è una figura immaginaria che rimane intessuta nelle forme schematiche e stereotipiche costitutive di alcuni dei modi di pensare abituali che sottendono invariabilmente ai tentativi delle scienze sociali di affrontare le questioni relative alla modernità politica dell'Asia meridionale. In qualunque parte del mondo ci troviamo, è impossibile pensare il fenomeno della "modernità politica" - la presenza di istituzioni moderne quali lo Stato, la burocrazia e l'impresa capitalistica - senza ricorrere a categorie e concetti che affondano le radici nelle tradizioni intellettuali, e perfino teologiche, europee ${ }^{3}$.

${ }^{1}$ Provincializing Europe. Postcolonial Thought and Historical Difference, Princeton University Press, Princeton-Oxford 2000. La traduzione italiana, priva del sottotitolo e dell'indice dei nomi, è apparsa a Roma, per Meltemi, nel 2004: tutte le citazioni saranno tratte da quest'ultima versione; avverto però che un riscontro costante con l'originale è sempre necessario.

${ }^{2}$ Ivi, p. 335.

${ }^{3}$ Ivi, p. 16. Corsivo dell'autore. 
N.B: Copia ad uso personale. È vietata la riproduzione (totale o parziale) dell'opera con qualsiasi mezzo effettuata e la sua messa a disposizione di terzi, sia in forma gratuita sia a pagamento.

Il Concilio Vaticano II e la "contestazione" religiosa del successivo sono parte del fenomeno della "modernità politica"? Attraverso quali tradizioni intellettuali, e perfino teologiche, europee sono pensabili? Il mio esercizio di lettura muove dalle domande appena formulate e non appartiene al genere della storia della storiografia, bensì a quello della storia della ricerca storica, che implica

un'analisi sia teorico-problematica sia critico-filologica delle modalità per cui e secondo cui uno studioso ha condotto la sua ricerca e infine ha conseguito certi risultati fattuali attraverso la documentazione e li ha pensati arricchendoli "di senso" con la propria operazione ermeneutica: una "interpretazione di senso" di "ciò che è realmente accaduto" nell'azione e nel pensiero degli uomini ${ }^{4}$.

Il testo da cui partirò è uno dei più significativi della stagione postconciliare, per l'America Latina e l'Italia. Teologia della liberazione di Gustavo Gutiérrez apparve in italiano nel 1972, come undicesimo volume della Biblioteca di teologia contemporanea dell'editore bresciano Queriniana ${ }^{5}$. Fino ad allora, la collana aveva ospitato contributi dei più noti teologi legati all'esperienza del Vaticano II (Johann Baptist Metz, Hans Küng, Joseph Ratzinger, Jürgen Moltmann, Piet Schoonenberg), radicati in Europa, tra Germania e Olanda. Non erano mancati due contribuiti estranei a questa geografia nordeuropea (con il saveriano italiano Battista Mondin e il gesuita spagnolo Juan Alfaro), ma fu il libro di Gutiérrez (nato a Lima, in Perù, nel 1928, prete diocesano prima, frate predicatore poi) a segnare un punto di svolta. Nel 1971-1972 divenne chiaro che cosa fosse la liberazione:

uomini e donne combattono oggi per la liberazione da ogni ingiustizia ed oppressione. I cristiani, partecipi di quest'ansia e di quest'impegno, di fronte all'ampiezza dei problemi connessi con il processo di liberazione, s'interrogano sul significato stesso del cristianesimo e sulla missione della chiesa, sul loro senso di fronte a una responsabilità umana tanto urgente. Questo libro co-

${ }^{4}$ G.G. Merlo, Conclusioni [a chiusura degli atti del convegno Medioevo e modernismo, organizzato da Umberto Longo e Francesco Mores presso l'Istituto storico italiano per il medio evo di Roma, 22 aprile 2016], in «Bullettino dell'Istituto storico italiano per il medioevo», 2018, 120, p. 342.

${ }^{5}$ Il volume reca un sottotitolo secco: Prospettive. La traduzione fu condotta sulla versione spagnola stampata a Roma nello stesso anno, presso Idoc, ma la prima edizione apparve come Cep, Lima 1971. D'ora in avanti, citerò la traduzione italiana con la sigla "TL". 
N.B: Copia ad uso personale. È vietata la riproduzione (totale o parziale) dell'opera con qualsiasi mezzo effettuata e la sua messa a disposizione di terzi, sia in forma gratuita sia a pagamento.

stituisce una riflessione, fondata sull'evangelo e sull'esperienza concreta di gruppi di cristiani dell'America latina, che può riuscire esemplare ed illuminante anche per chi vive in contesti differenti ${ }^{6}$.

Quanto alla teologia, nelle pagine di apertura del suo volume Gutiérrez tracciò una linea di sviluppo che è forse la parte meno letta del libro, centrata sulla necessità di stabilire in via preliminare quale sia «la funzione critica della teologia in rapporto alla presenza e all'azione dell'uomo nella storia» ${ }^{7}$. Secondo Gustavo Gutiérrez, la «riflessione teologica» sarebbe una sorta di frutto spontaneo della coscienza di ciascun credente, simile a ciò che per Antonio Gramsci era la filosofia: propria di tutti gli uomini, e non solo di una determinata categoria di specialisti ${ }^{8}$. Distinta da essa, «la prospettiva del lavoro teologico» era un dato non secondario della storia della Chiesa. Nei primi secoli di questa storia, lo «sforzo per un'intelligenza della fede» aveva assunto i caratteri di una ricerca sapienziale: «Si trattava, fondamentalmente, di una meditazione sulla Bibbia, fatta per progredire spiritualmente; si faceva distinzione fra i 'principianti', semplici fedeli, e i 'progrediti' che cercano la perfezione» ${ }^{9}$. I monasteri erano i luoghi deputati a tale ricerca, dove si pregava e si dialogava con parte del pensiero antico. Tutto ciò era durato fino al XII e XIII secolo, quando - riecheggiando Gutiérrez gli studi del frate predicatore Marie-Dominque Chenu ${ }^{10}-$ la teologia aveva cominciato un cammino che la condusse a prendere anche la forma di una scienza. Con Alberto Magno e soprattutto Tommaso d'Aquino, la teologia crebbe come «disciplina intellettuale frutto dell'incontro tra la fede e la ragione», un vero e proprio «sapere razionale» che prese la mano a coloro che la praticarono e che, a partire dal XIV secolo, la trasformarono in un sistema chiuso ${ }^{11}$. Per l'autore della Teologia della liberazione,

${ }^{6}$ Risvolto di copertina (redazionale?) di TL.

7 TL, p. 7.

${ }^{8}$ TL, p. 11 e, per il riferimento a Gramsci, ivi, nota 1 (dal centone La formazione dell'uomo. Scritti di pedagogia, a cura di G. Urbani, Editori Riuniti, che Gutiérrez cita nella seconda edizione apparsa a Roma nel 1969). La citazione seguente è tratta ancora da p. 11.

${ }^{9}$ TL, p. 12.

${ }^{10}$ Citato esplicitamente in TL, nota 12 p. 13: M.D. Chenu, La théologie est-elle une science?, Fayard, Paris 1957 (trad. it. Paoline, Catania 1958). Sull'importanza di Chenu per Gutiérrez, fin dagli anni trascorsi a Lione per completare la sua formazione teologica (1955-1959), si veda L. Ceci, La teologia della liberazione in America Latina. L'opera di Gustavo Gutiérrez, FrancoAngeli, Milano 1999, pp. 25 e 50; più in generale, a modo di introduzione a Teologia della liberazione. Prospettive, si vedano nello stesso volume le pp. 154-177.

${ }^{11}$ TL, p. 14. 
N.B: Copia ad uso personale. È vietata la riproduzione (totale o parziale) dell'opera con qualsiasi mezzo effettuata e la sua messa a disposizione di terzi, sia in forma gratuita sia a pagamento.

il sistema della Scolastica venne davvero meno con Giovanni XXIII e col Concilio Vaticano II, quando cominciò a definirsi una teologia dei segni dei tempi. La costituzione Gaudium et spes spiegava ai teologi quella che era la loro funzione: «contribuire ad una maggiore chiarezza in questo impegno, grazie ad un'analisi intellettuale» ${ }^{12}$.

L'analisi è - o dovrebbe essere - propria anche degli studiosi che si occupano delle geografie della contestazione. Il discorso storico sulla contestazione non gode di uno statuto di eccezione, tanto da rendere difficile comprendere che cosa si intenda con tale termine. Prima della serie di eventi e mutamenti riuniti sotto il nome collettivo "1968", l'uso della parola contestazione rientrava ancora nella sfera del diritto: in un'aula di tribunale, durante un processo, le parti in causa contestavano ufficialmente, le une alle altre, i termini di una controversia. Oggi, a cinquant'anni di distanza, la contestazione per antonomasia non è più un atto processuale: è un aspetto connotante del lungo "1968", con il quale si indica un movimento di critica e opposizione delle società uscite dal Secondo conflitto mondiale. In forma molto più accentuata del termine dissenso (con cui continuiamo a indicare una divergenza di opinioni, di sentimenti, di interessi), l'uso di contestazione mette in evidenza uno dei problemi che il mio esperimento di lettura pone: quando e perché il Vaticano II e la sua ricezione si saldarono con il tema della contestazione?

Una risposta secca è impossibile. Nell'ultimo quarantennio contestazione e dissenso sono stati utilizzati in modo oscillante, senza preoccuparsi troppo di definirne con chiarezza il significato. Questa indistinzione è, mi pare, strettamente legata al fatto che, nello studio delle geografie della contestazione, molti studiosi di storia si sono comportati da storici, lasciando che la propria biografia e le proprie esperienze di vita e politiche condizionassero la scelta dell'oggetto di studio. Se le cose stanno così, fare storia della ricerca storica sulle geografie della contestazione negli ultimi quarant'anni sarà molto più utile di un'analisi condotta anche con i criteri della storia della storiografia, dal momento che quest'ultima «considera soltanto il prodotto finale indipendentemente dalla fondatezza e verificabilità dei suoi contenuti, mentre la storia della ricerca storica guarda al problema da cui lo studioso muove e al percorso seguito - seguito attraverso il deposito e il territorio documentari - e, nel medesimo tempo, ai mezzi e alle tecniche usati per giungere alla tesi finale, di cui occorre accertare la fondata attendibilità prima di tutto documentaria e poi logico-concettuale» ${ }^{13}$.

${ }^{12}$ TL, p. 18.

${ }^{13}$ Merlo, Conclusioni, cit., p. 342. 
N.B: Copia ad uso personale. È vietata la riproduzione (totale o parziale) dell'opera con qualsiasi mezzo effettuata e la sua messa a disposizione di terzi, sia in forma gratuita sia a pagamento.

Alla luce della storia della ricerca storica e del problema della biografia, anche la più recente e accurata rassegna sulla storia del dissenso cattolico acquista nuovi significati. Nella messa a punto di Alessandro Santagata ${ }^{14}$, la storiografia sul dissenso è articolata in tre momenti, dalle prime riflessioni e i primi studi (1969-1977), attraverso gli studi più maturi (19781988), fino alla bibliografia recente (1989-2005); la cornice all'interno della quale la suddivisione è impostata è altrettanto importante della tipologia del dissenso esaminato: «senza negare cittadinanza in questo discorso ai tanti protagonisti nella contestazione nella Chiesa, in questa rassegna sarà privilegiata l'attenzione degli studiosi verso le forme di dissidenza dal "basso", quelle dei laici» ${ }^{15}$. Alto e basso sono categorie niente affatto neutre, non diversamente da destra e sinistra; dai tempi del modello duale della Storia naturale della religione di David Hume (analizzato - mostrandone tutti i limiti e la forza costantemente pervasiva - da Peter Brown ormai più di quarant'anni fa $)^{16}$, che cosa stia in alto e che cosa in basso è soprattutto legato al punto di vista dell'osservatore: se riportiamo questa considerazione al problema della storiografia sulla contestazione, saremo costretti ad aggiungere almeno un piano all'edificio costruito grazie alla tensione fra chierici (in alto) e laici (in basso), e questo piano è stabilmente occupato dagli ex-chierici. I quattro decenni presi in esame da Santagata possono essere riesaminati sulla base della mappa appena tracciata, a partire dal volume di Carlo Falconi (ex-chierico) La contestazione nella Chiesa, pubblicato a Milano nel $1969^{17}$; pochi mesi più tardi, ancora a Milano, fu un altro ex-sacerdote, Arnaldo Nesti, ad allestire l'antologia L'altra Chiesa in Italia ${ }^{18}$. Quattro anni dopo, nel 1974, sarà invece un chierico, Lorenzo Bedeschi, a offrire uno dei primi saggi sul tema, considerando nel suo Cattolici e comunist ${ }^{19}$ uno degli aspetti che verranno trattati dal piccolo libro del padre gesuita Giacomo Martina sulla Chiesa in Italia negli ultimi trent'anni, pubblicato in prima edizione nel febbraio $1977^{20}$. Il libro di Martina è già una sorta di bilancio - pubblicato in un momento che è

14 A. Santagata, Una rassegna storiografica sul dissenso cattolico in Italia, in «Cristianesimo nella storia», 2010, 31, pp. 207-241.

${ }^{15}$ Ivi, p. 207.

${ }^{16}$ P. Brown, Conoscenza e immaginazione, in Id., La società e il sacro nella tarda antichità, Einaudi, Torino 1988 (ed. or. University of California Press, Berkeley 1982), pp. 10-11.

${ }^{17}$ C. Falconi, La contestazione nella Chiesa, Feltrinelli, Milano 1969.

${ }_{18}$ A. Nesti, L'altra Chiesa in Italia, Mondadori, Milano 1970.

${ }^{19}$ L. Bedeschi, Cattolici e comunisti. Dal socialismo cristiano ai cristiani marxisti, Feltrinelli, Milano 1974.

${ }^{20}$ G. Martina, La Chiesa in Italia negli ultimi trent'anni, Studium, Roma 1977. 
N.B: Copia ad uso personale. È vietata la riproduzione (totale o parziale) dell'opera con qualsiasi mezzo effettuata e la sua messa a disposizione di terzi, sia in forma gratuita sia a pagamento.

l'inizio dell'«inizio della fine dei movimenti di protesta» e «del fallimento delle proposte di alternativa al modello politico ed ecclesiologico dominante partorite dalla contestazione stessa $»^{21}-$ a cui, nella prima metà degli anni ottanta, fece seguito quella sorta di bilancio (esistenziale) del bilancio (bibliografico) che è il Dissenso cattolico in Italia di Mario Cuminetti ${ }^{22}$, un'opera che fa da spartiacque nel saggio di Santagata. Dopo il 1983, e tanto più nella bibliografia recente, non troveremo più biografie così significative come quella di Cuminetti: sacerdote, storico della teologia evangelica e riformata, presente al concilio al seguito del vescovo di Segni Luigi Carli, assistente ecclesiastico dell'Augustinianum della Cattolica dal 1966 al 1967, nel 1968 assistente di Witte in Gregoriana per l'insegnamento di Teologia ecumenica, nel 1972 assunto alla libreria della Corsia dei servi, nel 1973 membro della segreteria dei Cristiani per il socialismo (anno in cui chiese e ottenne la riduzione allo stato laicale).

La scomparsa di simili biografie dal nostro orizzonte storiografico rafforza alcuni dei nodi che ho cercato di mettere in luce finora: 1) la centralità del nesso tra biografia, storiografia e autobiografia; 2) la necessità di definire con chiarezza gli usi di contestazione e dissenso, nei loro rapporti con la parola protesta. Per provare a sciogliere questi nodi, ho scelto due momenti della storiografia sul tema della contestazione, ai due estremi del quarantennio che va dal 1977 al 2017, trasformandoli in due momenti della mia riflessione. Nel primo sono stato più preciso, nel secondo meno (pure di fronte a opere recentissime che entrano nel vivo del problema che affronto qui) ${ }^{23}$. La ragione di quest'ultimo slittamento è legata alla dimensione geografica. Credo sia impossibile comprendere che cosa sia davvero avvenuto in Italia negli anni della "contestazione" e dell'onda lunga del Concilio Vaticano II senza allargare il punto di vista all'America latina e al medioevo, termine che traduce in maniera più puntuale la questione del rapporto tra l'azione nel presente e il passato.

Non la storia, si badi, ma il passato. Il fatto che Teologia della liberazione di Gustavo Gutiérrez sia stato tradotto in italiano da una delle figure più significative dell'esperienza dei preti operai, Luisito Bianchi, non è solo un aneddoto, ma è la conferma di passaggi importanti del libro di Gutiérrez come quello che segue:

${ }^{21}$ Santagata, Una rassegna storiografica, cit., p. 207.

${ }^{22}$ M. Cuminetti, Il dissenso cattolico in Italia, 1965-1980, Rizzoli, Milano 1983.

${ }^{23}$ S. Inaudi, M. Margotti (a cura di), La rivoluzione del Concilio, La contestazione cattolica negli anni Sessanta e Settanta, Studium, Roma 2017. 
N.B: Copia ad uso personale. È vietata la riproduzione (totale o parziale) dell'opera con qualsiasi mezzo effettuata e la sua messa a disposizione di terzi, sia in forma gratuita sia a pagamento.

attorno al XIV secolo inizia una separazione fra teologi e spirituali. Troviamo questa separazione, ad esempio, in un libro come L'imitazione di Cristo, che segnò fortemente la spiritualità cristiana in questi ultimi secoli. Ancora oggi soffriamo di questa separazione, anche se è certo che il rinnovamento biblico e la necessità di riflettere sulla spiritualità dei laici stanno già dando segni di quella che può considerarsi una nuova teologia spirituale ${ }^{24}$.

Molti dei libri sulle esperienze di liberazione dell'America latina contenevano anche passi come quello appena riportato, che connettevano il medioevo europeo (a partire dal De civitate Dei di Agostino) fino a quella che allora, agli inizi degli anni settanta del Novecento, era la contemporaneità, segnata da testi come la Gaudium et spes e dalla teologia dei segni dei tempi. La teologia della quale parlava Gutiérrez era sia spirituale, sia un sapere razionale ${ }^{25}$, riguardava chierici e laici, faceva riferimento all'America latina e guardava al passato europeo, servendosene per interpretare il presente. Nei due paragrafi che seguono proveremo a interpretare il presente del 1977 e intorno al 2017 anche grazie a ciò che fu pensato in America latina. Sarà dunque un esercizio di provincializzazione, che terrà insieme tempi e luoghi molto lontani fra loro.

\section{7}

Come è noto, tra il 22 e il 24 settembre 1977 sì riunì a Bologna un convegno che, a più di quarant'anni di distanza, continua a essere definito "sulla repressione". Poche settimane prima o poche settimane dopo, tra le stesse vie e piazze, un medievista e storico della Chiesa romana, Ovidio Capitani, scrisse un'introduzione a un'antologia di studi sul Medioevo ereticale con gli occhi fissi a quello che allora era il passato prossimo o il futuro anteriore. Quelle di Capitani non furono affatto - o non furono solo - analisi per gli addetti ai lavori, bensì «riflessioni più generali su modi e forme della protesta/devianza e sul suo farsi (o non farsi) motore di storia $»^{26}$. Ne riporto uno dei passaggi più importanti:

non certo che la devianza religiosa [...] sia un fatto di poco conto, che possa essere trascurato o liquidato; ma un fatto che non può essere colto soltanto $\mathrm{o}$

${ }^{24}$ TL, p. 13.

25 TL, p. 15.

${ }^{26}$ G.G. Merlo, Intorno ad eretici ed eresie, in Id., Eretici del medioevo. Temi e paradossi di storia e storiografia, Morcelliana, Brescia 2011, p. 91. 
N.B: Copia ad uso personale. È vietata la riproduzione (totale o parziale) dell'opera con qualsiasi mezzo effettuata e la sua messa a disposizione di terzi, sia in forma gratuita sia a pagamento.

prevalentemente nei suoi elementi di “protesta” contro l'istituzione. Assumere questa dimensione interpretativa - anche se per valutare positivamente quella protesta, anche se per privilegiarla e magari trarne suggestioni "retoriche" per una pubblicistica contemporanea - è collocarsi nella prospettiva angusta in cui fatalmente sono collocati gli "eretici" dalla pubblicistica e dalla libellistica di parte che li perseguita e li dipinge come segni "negativi" rispetto al "positivo" di una sistemazione molto sofisticata della religiosità, della cultura teologica avanzata [...]. Solo accettando per pacifico il divario larghissimo tra la cultura e l'articolazione razionale del "sistema" teologico-giuridico inquisitoriale e le credenze e gli atteggiamenti confusamente devozionali, $\mathrm{o}$, al più, contraffattori della stessa istituzione ecclesiastica [...], solo scontando appunto la "marginalità" del fatto religioso, dell'incidenza religiosa nell'insieme del fenomeno della devianza si può recuperare il vero volto dell'eretico. E non perché l'aspetto religioso sia una sovrastruttura [...], ma perché quell'aspetto religioso che non sappia farsi istituzione (ovviamente non in senso meramente normativistico, ma sì storicistico), che non sappia magari mutare profondamente l'istituzione - ma deve legarsi a forze operanti nella storia, non efficaci sul piano di attese esclusive - non solo è destinato ad essere - come fu - perdente, ma è condannato a non dirci nulla di quello che era l'oggetto del rifiuto e del perché del rifiuto, della devianza ${ }^{27}$.

Osservata da un punto di vista interno alla storia della Chiesa cattolicoromana, con un accento particolare sull'età medievale, la definizione di dissenso/protesta/contestazione acquista alcune caratteristiche peculiari: non può essere limitata alla semplice protesta contro l'istituzione e non può essere racchiusa nella categoria spesso giornalistica di eresia, né essere osservata solo dal punto di vista di chi giudica i dissenzienti come tali, ponendoli in una condizione di minorità rispetto a un sistema ecclesiastico raffinato, che proprio per la sua raffinatezza non può accettare la contestazione; all'interno e all'esterno del sistema vi è il fatto religioso, ma l'incidenza dell'esterno sull'interno è legata alla capacità del movimento di farsi istituzione. Negli altri cattolicesimi politici c'è stato sicuramente anche questo, ma non va trascurato il fatto che, forse, vi furono anche altri cattolicesimi e, in alcuni casi, altri cristianesimi. Si pensi, per esempio, al già ricordato patrimonio di idee che una casa editrice cattolica come la Queriniana di Brescia mise a disposizione dei suoi lettori, al di là della $B i$ blioteca di teologia contemporanea in cui, nel marzo del 1972, fu stampato Teologia della liberazione di Gustavo Gutiérrez; nel 1996 Lucia Ceci ha ricostruito la parabola della Queriniana attraverso il decennio successivo

${ }^{27}$ O. Capitani, Introduzione [settembre 1977?], in Medioevo ereticale, a cura di Id., il Mulino, Bologna 1977, pp. 14-16. 
N.B: Copia ad uso personale. È vietata la riproduzione (totale o parziale) dell'opera con qualsiasi mezzo effettuata e la sua messa a disposizione di terzi, sia in forma gratuita sia a pagamento.

alla chiusura del Concilio Vaticano II, fino alla fine degli anni settanta e al caso Hans Küng, mostrando la particolare incidenza che, attraverso la traduzione, in essa hanno avuto gli altri cristianesimi, e in particolare la teologia evangelica e riformata ${ }^{28}$. Le opere di figure divenute subito di rilievo, le storie delle altre teologie e soprattutto i testi, che potevano dischiudere un mondo altro (ma pur sempre cristiano): è il caso della collana del $D i$ partimento di scienze religiose allora ospitata da Queriniana, dove nel 1977 apparvero la raccolta di Giuseppe Alberigo sulla Riforma protestan$t^{29}$ e quella di Pier Cesare Bori sulla Chiesa primitiva ${ }^{30}$. Chi avesse voluto informarsi su una chiesa non ancora compromessa con il potere avrebbe trovato nel libro di Bori la guida per capire come andarono le cose. E come andarono davvero le cose lo si comprende fin da subito, dall'esordio dell'Introduzione all'antologia:

diciamo subito che in questa raccolta abbiamo preso come punto di partenza la chiesa (o le chiese) dell'epoca neotestamentaria e come termine ultimo pressappoco la metà del secolo, dando però rilievo soprattutto al secolo secondo e accogliendo invece gli ultimi documenti (Ippolito, Cipriano, Origene...) come testimonianze di una crisi che già anticipa la cosiddetta 'svolta costantiniana', come documenti di una trasformazione di cui gli autori stessi sono consapevoli, denunciando anzi il decadimento della chiesa in rapporto, appunto, all'epoca 'primitiva's1.

Credo che La chiesa primitiva sia un libro esemplare di molte delle linee-forza che attraversavano allora il problema del rapporto tra il Concilio e la contestazione. Bori si era formato all'Università cattolica e aveva studiato al Seminario lombardo, al Pontificio istituto biblico e alla Gregoriana di Roma; le svolte della sua biografia indicano uno sforzo volto anche a mettere a disposizione di un pubblico più vasto, interessato a servirsene, una definizione primaria di chiesa che contestasse il decadimento di una istituzione che veniva percepita come compromessa con il potere.

Su questo punto specifico, il 1977 aveva ancora molte cose da dire. In quell'anno maturarono opere pensate in un passato prossimo e riflessioni destinate al futuro anteriore. Ripartiamo dal passato prossimo. Il già ricordato profilo La Chiesa in Italia negli ultimi trent'anni di Giacomo Mar-

${ }^{28}$ L. Ceci, L'editoria cattolica nel periodo postconciliare. Il caso della Queriniana, in «Annali della Fondazione Luigi Einaudi», 1996, XXX, pp. 393-431 (alla fortuna delle opere di Gutiérrez, a partire da Teología de la liberación sono dedicate le pp. 414-415).

${ }^{29}$ G. Alberigo, La riforma protestante. Origini e cause, Queriniana, Brescia 1977.

${ }^{30}$ P.C. Bori, La chiesa primitiva, Queriniana, Brescia 1977.

${ }^{31}$ Ivi, p. 7. 
N.B: Copia ad uso personale. È vietata la riproduzione (totale o parziale) dell'opera con qualsiasi mezzo effettuata e la sua messa a disposizione di terzi, sia in forma gratuita sia a pagamento.

tina reca, al termine dell'introduzione, la data "luglio 1976", mese in cui Martina dovette terminare di rivedere quella che è una versione ampliata di una relazione presentata nel dicembre del 1974 a un seminario ristretto del Movimento laureati di Azione cattolica, stampata nel 1975 negli atti del convegno La Chiesa in Italia tra fede e storia. E dal titolo del convegno l'autore sembrò trarre uno schema interpretativo da applicare nei paragrafi dedicati alla contestazione e alla contro-contestazione ${ }^{32}$.

Secondo Martina, Comunione e Liberazione e il movimento carismatico furono "contro" nella misura in cui seppero contrastare i pericoli del soggettivismo (si tenga a mente questo termine, accanto ad altri sempre in corsivo) con - nel caso dei carismatici - «la prudente guida di alcuni sacerdoti (visti in genere con grande rispetto), e corsi sistematici di approfondimento teologico». Anche nel caso di $\mathrm{Cl}$, è il rapporto con i chierici (e la gerarchia) a fare da metro di giudizio. La parola impiegata, ripresa da un giudizio del padre Bartolomeo Sorge (gesuita come Giacomo Martina) ha una lunga storia nella Compagnia fondata da Ignazio di Loyola: metodo. Il rischio di valide «intuizioni di base» prive di discernimento era grave: non trovare «la via giusta per realizzare» i propri ideali, rischiando «di costituire un elemento di frattura e non di comunione, per il suo spirito massimalista», e cadendo "per timore di ogni compromesso [...] in un certo integrismo». Lo storico gesuita - noto soprattutto per i suoi studi su Pio IX e per il giudizio contrario alla beatificazione di papa Mastai - ammirava l'«indubbio coraggio» dei membri di $\mathrm{Cl}$ che, nelle Università, affrontavano le «violenze fisiche dei gruppi extraparlamentari, intolleranti di ogni propaganda per altre idee». Dal momento che La Chiesa in Italia negli ultimi trent'anni apparve in prima edizione nel febbraio del 1977, quest'ultimo inciso non può ricordare oggi l'episodio che, l'11 marzo 1977, ancora a Bologna, diede il via agli scontri di piazza durante i quali morì uno studente. Martina non poteva certo prevedere che il casus belli sarebbe stato proprio il tentativo di interrompere un'assemblea convocata da Comunione e Liberazione, ma la coincidenza resta significativa. La battaglia non solo di idee combattuta anche a Bologna creò la categoria di contro-contestazione che è vera come - l'esempio è di Martina - lo è la categoria di controriforma, indicando «solo una parte della verità»: non si trattò «tanto di opposizione alla contestazione, quanto di superamento di essa, non per negarne i motivi più validi per acquisirli in un contesto diverso, di fedeltà alla gerarchia e di accentuazione del momento propriamente religioso su quello politico».

${ }^{32}$ Martina, La Chiesa in Italia, cit., pp. 151-173; fino a diversa indicazione, tutte le citazioni nel corpo del testo saranno tratte da qui. 
N.B: Copia ad uso personale. È vietata la riproduzione (totale o parziale) dell'opera con qualsiasi mezzo effettuata e la sua messa a disposizione di terzi, sia in forma gratuita sia a pagamento.

Nella Chiesa in Italia negli ultimi trent'anni, questo giudizio arriva al termine di una lunga illustrazione della contestazione (connotata anche dalla violenza) e del dissenso (inteso, invece, come ricerca di una «larga autonomia nei confronti della gerarchia»), che hanno entrambi alla loro radice una condizione psicologica. La contestazione del 1967-1968 fu una rivoluzione mondiale, scoppiata quando «l'opinione pubblica acquista coscienza della propria depressione, quando cioè si attuano con ritardo e lentezza, le prime timide riforme». Il Vaticano II arrivò forse «troppo tardi» o, almeno, si tardò ad attuarlo, a causa di «incertezze, paure, scarsa creatività». Anche in tal caso, il rimedio indicato è quello della tradizione gesuitica: il discernimento, che avrebbe potuto aiutare a distinguere tra ciò che andava confermato e quanto andava mutato. Si badi che la priorità assegnata alla politica è solo un aspetto della più generale psicopatologia della contestazione. La descrizione della contestazione politico-sociale si scioglie nella «contestazione talora disumana di qualche determinato professore» e il passaggio al piano religioso costituisce «una conseguenza e un riflesso inevitabile di quella politica», ma con una sua specificità, ovvero l'avvertita distanza tra lo «spirito del Vangelo» e «un pesante apparato istituzionale che rischia di soffocare il carisma». Per Martina, la Chiesa cattolica aveva fino ad allora mancato della «fantasia» necessaria a riempire questo iato; alcuni cattolici avevano provato a colmarlo con il marxismo, ma - per una «valutazione globale» della contestazione - era ancora la psicologia a essere rilevante, tanto per i laici, quanto per l'istituzione ecclesiastica:

essa [la contestazione] è talora frutto di irrequietudine, di scontentezza, di frustrazioni personali. Conoscendo da vicino alcuni protagonisti, posso dire che essi trasferiscono qualche volta in altri cristiani problemi personali non risolti. E mi sarebbe facile, anche se non generoso, fare dei nomi. Ma sarebbe del tutto ingiusto ed antistorico valutare globalmente il fenomeno sulla falsariga di questi singoli casi. C'è piuttosto in più di uno una certa ingenuità ed intemperanza, una mancanza di spirito critico, il bisogno di un capo, di uno slogan. In altre parole, la generazione del dopoguerra, accanto a tanti lati positivi, come la sincerità, la generosità, lo spirito di sacrificio, nasconde sotto una maschera di sfrontatezza una reale insicurezza psicologica. I giovani avvertono spesso il bisogno di irreggimentarsi, senza sapere bene dove e per quali motivi. E proprio quest'insicurezza li rende facilmente estremisti, impazienti, facili a cedere alla violenza verbale, e talora anche fisica, e soprattutto incapaci di esercitare una vera critica, più disposti a distruggere che a costruire. Ma non possiamo nemmeno dimenticare i motivi più profondi e più oggettivi, l'aspirazione a una Chiesa più pura e più fedele al Vangelo, la sensibilità per l'urgenza di certe ri- 
N.B: Copia ad uso personale. È vietata la riproduzione (totale o parziale) dell'opera con qualsiasi mezzo effettuata e la sua messa a disposizione di terzi, sia in forma gratuita sia a pagamento.

forme, il rifiuto di troppi compromessi tuttora palesi. La contestazione insomma esiste perché la Chiesa ha ancora bisogno di una profonda riforma: reformata reformanda.

Il discernimento esercitato nel passo appena riportato è un punto centrale del metodo impiegato nella Chiesa negli ultimi trent'anni. Martina provò a descrivere i moti dell'anima (altro termine della tradizione gesuita, presente - come il metodo e il discernimento - negli Esercizi spirituali di Ignazio) della contestazione, ma non trascurò di dare un nome a quella fra le idee che egli riteneva più legata alla situazione allora presente: il soggettivismo. Onnipresente e inafferrabile, esso fu associato a due figure, che lo storico gesuita provò a leggere ricorrendo alla propria esperienza personale, di chierico e studioso, collegandole all'età che gli sentiva più sua, quella di Pio IX. Ecco come egli tratteggiò la prima figura (legata a un libro tradotto ancora dalla Queriniana):

nel '70 il libro di Hans Küng, Infallibile? Una domanda (Brescia, 1970, tr. dal ted.), presto esaurito, solleva gravi problemi sull'ermeneutica del dogma del 1870. Il lavoro del Küng, scientificamente affrettato e fondato troppo spesso su fonti di seconda mano e non privo di luoghi comuni, in parte si riallaccia alle tesi già affacciate dal Döllinger nelle sue Römische Briefe vom Konzil, presentazione unilaterale e tendenziosa della linea seguita da Pio IX, in parte solleva nuove e gravi questioni, senza dare ad esse una risposta esauriente.

\section{Ed ecco il secondo ritratto:}

storicamente, una critica esercitata senza moderazione, spinta a una contestazione di alcuni valori fondamentali, portata a sottovalutare la dimensione escatologica del Vangelo, irrigidita in più o meno una chiara rottura con l'istituzione, rischia di provocare quanto accadde con i preti liberali del secolo scorso, come il gesuita p. Curci ed il benedettino Simplicio Pappalettere (che sia detto per inciso, può essere considerato idealmente padre di don [in realtà dom] Franzoni, perché abate di S. Paolo e di Montecassino, deposto dal suo ufficio e sospeso a divinis per il suo atteggiamento liberale): un irrigidimento della gerarchia su posizioni conservatrici, una chiusura in luogo dell'apertura desiderata.

In una prospettiva di storia della ricerca storica, il ponte gettato fra il 1970 e il 1870 spiega molto del modo di lavorare Giacomo Martina. Il suo passato prossimo era fatto di categorie come controriforma e contro-contestazione e di problemi, primo fra tutti quello dell'infallibilità, discusso al Vaticano I. Senza Döllinger non vi sarebbe stato Küng, senza Simplicio 
N.B: Copia ad uso personale. È vietata la riproduzione (totale o parziale) dell'opera con qualsiasi mezzo effettuata e la sua messa a disposizione di terzi, sia in forma gratuita sia a pagamento.

Pappalettere, dom Giovanni Franzoni: credo che quest'ultima connessione sia la più esemplificativa del metodo fondato anche sul discernimento impiegato da Martina, non perché il liberalismo di Pappalettere abbia a che fare con le prese di posizione anche politiche di Franzoni (non ultimo il ruolo centrale nella comunità di base di San Paolo, dopo la scelta, compiuta nel 1973, di lasciare l'abbaziato, e la successiva riduzione allo stato laicale, raccontate - si faccia attenzione al verbo utilizzato - nei diari in pubblico di Franzoni) ${ }^{33}$, ma perché la parola "liberalismo" traduce quello che fu il vero problema di Martina, ovvero come il concetto di autorità e gerarchia abbia subito modifiche rilevantissime, anche sulla spinta del contesto, dal Vaticano I al Vaticano II.

Anche di tale passato prossimo si ragionò nel 1977, con una prospettiva aperta al futuro anteriore. Fu questa l'operazione compiuta da Guido Verucci in una relazione intitolata Religione e scelte politiche negli studi storici del secondo dopoguerra, presentata il 19 maggio 1977 al convegno organizzato a Roma dall'Associazione per lo studio dei fenomeni religiosi contemporanei e pubblicata nella seconda metà dell'anno seguente ${ }^{34}$. Si trattò di un esercizio di distanziamento, fatto in un anno non facile, chiuso dalla constatazione che, per i gruppi che contestavano in forme anche molto diverse la Chiesa romana e la Democrazia cristiana, si fosse da tempo entrati nella dialettica classica tra riforme e rivoluzione, attraverso una radicalizzazione delle prospettive politiche e religiose, dalla fine degli anni quaranta fino alla seconda metà degli anni settanta. Tra gli acceleratori di tale processo, troviamo quelli che diventeranno i cardini dell'analisi di Verucci sul problema: il Concilio Vaticano II, con le speranze e le delusioni che esso suscitò; le lotte internazionali degli anni settanta; la secolarizzazione; il rapporto - spesso confuso - con segmenti di un marxismo difficilmente afferrabile; l'atteggiamento assunto dal Pci verso la questione religiosa. Vi è poi un ulteriore scintilla, che andrà chiarita alla luce di una citazione più ampia che farò subito. Come si spiega l'emersione «di nuove forme di religiosità popolare evangelico-politica, in cui il rapporto fra religione e politica è analogo, anche se meno mediato, a quello di gruppi politici ed ecclesiali culturalmente più elevati»? Non so come intendere esattamente questo uso di "alto" e "basso", ma mi pare che la traiettoria del discorso di Verucci converga verso il punto che segue: 2014

${ }^{33}$ G. Franzoni, Autobiografia di un cattolico marginale, Rubbettino, Soveria Mannelli

${ }^{34} \mathrm{G}$. Verucci, Religione e scelte politiche negli studi storici del secondo dopoguerra, in «Belfagor», 1978, XXXIII, pp. 343-356. 
N.B: Copia ad uso personale. È vietata la riproduzione (totale o parziale) dell'opera con qualsiasi mezzo effettuata e la sua messa a disposizione di terzi, sia in forma gratuita sia a pagamento.

nelle espressioni estreme del movimento dei "Cristiani per il Socialismo", come in quelle di altri gruppi, la rottura con il cattolicesimo, anzi con il cristianesimo storico, che comporta la rottura più o meno esplicita e più o meno completa con la trascendenza e con il dogma come connotati della tradizione cristiana, si collega con la tendenza, suggerita da certi studi storici e teologici, a sottolineare in chiave più "mondana", rispetto alla tradizione, gli aspetti soteriologici ed escatologici del cristianesimo primitivo; si collega con la persistenza di una scelta esistenziale, a favore di motivi come quelli dell'amore, dell'uguaglianza, della giustizia, che hanno nel messaggio cristiano una rilevanza centrale e una ispirazione particolare. Se questa analisi è corretta, non si vede perché questi motivi non possano costituire la "specificità" dell'apporto dei cristiani alla lotta per il socialismo ${ }^{35}$.

Non posso dimostrare che le conclusioni di Verucci furono pensate come un dialogo a distanza con libri come La chiesa primitiva di Bori: l'accenno a una lettura mondana degli aspetti soteriologici ed escatologici del cristianesimo primitivo non basta. Ma ciò che va sottolineato è la volontà di andare al di là delle definizioni, per raggiungere la specificità di un fenomeno legato all'operazione interpretativa, che solo così acquista senso (senza ricorrere, lo si noti bene, alle parole dissenso, contestazione o protesta). Sullo stesso piano si era mosso Martina, stabilendo una connessione tra gli anni settanta dell'Ottocento e del Novecento e cercando di interpretare anche psicologicamente quanto avvenuto tra il 1967 e il 1977. E da parte sua Ovidio Capitani, nelle settimane del "settantasette" bolognese, aveva chiarito i termini del problema: le «attese esclusive» non mutano le cose e ancor meno creano o riformano istituzioni. Il contributo individuato da Verucci era dunque destinato a essere spazzato via. E cominciò forse a essere travolto nel momento in cui si fece rinchiudere nelle definizioni di dissenso, contestazione e protesta.

Non è caso, mi pare, che tali definizioni non compaiano in un altro dei libri importanti tradotti da Queriniana. Pubblicato in Brasile nel 1976 e in Italia nel 1977, Teologia della cattività e della liberazione del frate Minore Leonardo Boff (una delle figure di punta fra i "teologi della liberazione") ${ }^{36}$

${ }^{35}$ Ivi, p. 355.

${ }^{36}$ L. Boff, Teologia della cattività e della liberazione, Queriniana, Brescia 1977 (ed. or. Petrópolis-RJ 1976). Secondo Lucia Ceci (L'editoria cattolica, cit., p. 416), il libro sarebbe espressione del ridimensionamento degli «entusiasmi inziali» della teologia della liberazione, che lasciarono il posto «a una riflessione più pacata sul significato e le ragioni che stavano alla base dell'ostilità che i movimenti ecclesiali che si riconoscevano in tale prospettiva teologica incontravano nella società e nella Chiesa latinoamericana». Vale la pena riportare anche il periodo immediatamente successivo: «un'analoga disillusione si 
N.B: Copia ad uso personale. È vietata la riproduzione (totale o parziale) dell'opera con qualsiasi mezzo effettuata e la sua messa a disposizione di terzi, sia in forma gratuita sia a pagamento.

evita di fermarsi su categorie attribuite per rilanciare la prospettiva della liberazione attraverso quella della cattività. Muovendo dall'oppressione e dall'esodo del popolo ebraico in Egitto, Boff mise in moto una sua peculiare «ermeneutica degli avvenimenti storici $»^{37}$, che arriva alle soglie dell'età moderna:

la storia recente del Brasile è in continuità con il suo passato e può essere narrata con tutti i suoi morti. La colonizzazione iberica nel resto del Continente latino-americano non è stata meno violenta e repressiva. La storiografia ufficiale magnifica la conquista e i conquistatori; non dà ascolto alla storia e ai gemiti dell'autoconquista, di coloro che hanno sofferto la violenza e la depredazione in modo tanto inumano che in cinquant'anni di colonizzazione iberica si è provocata una ecatombe demografica che ha ridotto a meno della quinta parte la popolazione autoctona. Il grande difensore degli indios, il vescovo Bartolomeo de Las Casas descrive nel 1552 nella sua brevissima relazione sulla distruzione delle Indie i due modi di comportamento dei conquistatori in relazione all'indio: "uno basato su guerre ingiuste, crudeli, sanguinose e tiranniche; l'altro sull'uccisione di coloro che potevano anelare, sospirare o pensare alla libertà o alla soluzione dei tormenti che soffrivano, come fossero i padroni naturali degli uomini adulti (perché in guerra generalmente lasciano in vita solo donne e bambini) e opprimerli con la più dura, orribile e aspra schiavitù sotto la quale né uomini, né animali potettero mai essere posti ${ }^{38}$.

Vale la pena ricordare che uno degli ultimi volumi di Gustavo Gutiérrez è dedicato proprio a Bartolomé de Las Casas ${ }^{39}$. Riprendendo Leonardo Boff, potremmo rilevare come la storia recente sia pensata in continuità con il suo passato e possa essere narrata. Quest'ultimo verbo - narrare vale anche per la via d'uscita intravista da Boff per uscire dalla situazione venutasi a creare nella seconda metà degli anni settanta:

è a questo punto della conversione a livello strutturale che incide il contributo principale del cristianesimo. Esso afferma che l'uomo nuovo emergerà alla condizione e nell'esatta misura che tanto l'intimo dell'uomo che le strutture

ritrovava, sia pure con tratti e per motivazioni diverse, nel mondo cattolico italiano, soprattutto in quei settori che, dopo il Concilio, avevano riposto forti speranze nella possibilità di ripensare l'identità del cristianesimo e della Chiesa in modo più autentico e vicino ai valori evangelici». Come vedremo immediatamente, la mia prospettiva è in parte diversa.

${ }^{37}$ Ivi, p. 81 e, più diffusamente, pp. 116-122.

${ }^{38}$ Ivi, p. 134.

${ }^{39}$ G. Gutiérrez, Alla ricerca dei poveri di Gesù Cristo. Il pensiero di Bartolomé de Las Casas, Queriniana, Brescia 1995 (Biblioteca di teologia contemporanea, 80). L'edizione originale apparve in spagnolo per l'Instituto Bartolomé de Las Casas/Ceo, Lima 1992. 
N.B: Copia ad uso personale. È vietata la riproduzione (totale o parziale) dell'opera con qualsiasi mezzo effettuata e la sua messa a disposizione di terzi, sia in forma gratuita sia a pagamento.

sociali si convertano verso un altro progetto storico, qualitativamente differente da quello che caratterizza la modernità. Non più essere solamente sulle cose e sugli altri, ma stare principalmente con esse e con gli altri, in una radicale dimensione amorosa e fraterna ${ }^{40}$.

Solo dalla nota al passo appena riportato apprendiamo che il modello di questo nuovo progetto storico, diverso dalla modernità allora presente, è Francesco d'Assisi ${ }^{41}$. Nel secolo della trasformazione della teologia in una scienza (secondo il giudizio del padre Chenu sul XII e XIII secolo), l'Assisiate avrebbe dunque gettato un ponte tra il passato e il presente. Non attraverso la storia, si badi, ma nel passato. Il fatto che già la Teologia della liberazione di Gustavo Gutiérrez avesse intravisto questa possibilità, rafforza il fatto che il confronto tra due diverse geografie della contestazione (e le loro traduzioni, dall'America Latina all'Europa) possa aiutare a comprendere che cosa accadde alla fine degli anni settanta. L'invenzione del passato, trasformato in storia, poteva essere una via di uscita dalle categorie di dissenso, contestazione e protesta.

2017-

Le cose stanno ancora così, dopo quarant'anni? Ciò che è sicuro è che il tempo trascorso dal 1977 non ha scalfito l'importanza, per il contesto italiano, della riflessione di Guido Verucci, con in più la coscienza del filtro posto dalla documentazione e il problema della profondità. Nel suo libro del 2016, La contestazione cattolica ${ }^{42}$, Alessandro Santagata è partito da una considerazione contenuta nel saggio forse più noto di Verucci sul tema - Il dissenso cattolico in Italia, apparso nel $2002^{43}$ - per colmare la distanza che separa la fine del Vaticano II al 1968, definito «un momento di forte accelerazione e di radicalizzazione delle attese di riforma sollevate nel Concilio» ${ }^{44}$. La progressione del libro può essere spiegata anche così: si parte molto piano (con il capitolo La Conferenza episcopale e la ricezione del Concilio) e si giunge all'Esplosione della protesta, lungo una curva crescente che è anche quella del rinnovamento degli studi sul tema.

${ }^{40}$ Boff, Teologia della cattività, cit., p. 159.

${ }^{41}$ Ivi, nota 15 p. 159, con un rinvio a L. Boff, A não-modernitate de São Francisco, in «Vozes», 1975, 69, pp. 335-348.

${ }^{42}$ A. Santagata, La contestazione cattolica. Movimenti cultura e politica dal Vaticano II al '68, Viella, Roma 2016.

${ }^{43} \mathrm{G}$. Verucci, Il dissenso cattolico in Italia, in «Studi storici», 2002, 43, pp. 215-233.

${ }^{44}$ Santagata, La contestazione cattolica, cit., p. 15. 
N.B: Copia ad uso personale. È vietata la riproduzione (totale o parziale) dell'opera con qualsiasi mezzo effettuata e la sua messa a disposizione di terzi, sia in forma gratuita sia a pagamento.

Il punto zero della curva va collocato nel 2005, quando Morcelliana pubblicò Dal Concilio alla contestazione. Riviste cattoliche negli anni del cambiamento (1958-1968) di Daniela Saresella: in esso convergono lo studio del principale veicolo delle idee della contestazione (i periodici) con un allargamento dell'orizzonte ad archivi privati fino ad allora inesplorati. Le implicazioni di questa convergenza diventano chiare se si considera l'autore di un libro di riferimento già menzionato, Mario Cuminetti. Nel 1988, cinque anni dopo la pubblicazione del Dissenso cattolico, Cuminetti stese e forse non spedì una lettera in cui descrisse le occupazioni della Cattolica nel 1967 come il frutto di problemi organizzativi e di una «maturazione della coscienza dei cattolici provocata e accelerata dal Concilio» ${ }^{45}$. Anche in questo caso, come per Verucci, l'accelerazione conciliare era un elemento non trascurabile nella parabola della contestazione.

Sull'estensione di essa credo sia necessario fare chiarezza. Se accettiamo l'idea che il Vaticano II abbia rappresentato una nuova consapevolezza del posto della Chiesa romana nella storia, oscuriamo forse una parte del problema. Il problema non sta nella storia, ma nell'uso del passato: alcuni degli studi apparsi dopo il 2005 e prima del 2011 sembrano dimostrarlo.

Nel 2011 ancora Daniela Saresella ha inserito il problema della contestazione all'interno di una ricerca che rivela come il problema debba essere letto tenendo conto anche della profondità. Così, i Cristiani per il Socialismo potranno essere compresi solo tenendo presente che «questi credenti, al pari dei modernisti di inizio Novecento, pensavano che un rinnovamento della società non potesse prescindere da una riforma della Chiesa ${ }^{46}$. Allo stesso modo chi, morto nel 2008, vide nel 2010 raccogliere i suoi ultimi scritti su Chiesa cattolica ed esperienza religiosa ${ }^{47}$, dirà ai lettori di oggi molte più cose se essi saranno avvertiti che i suoi primi scritti apparvero su «L'uomo» di Apollonio, De Piaz, Turoldo, Romanò e che la sua casa paterna di Milano era frequentata da Antonio Aiace Alfieri, direttore, con Tommaso Gallarati Scotti e Alessandro Casati, del «Rinnovamento» ${ }^{48}$.

Non apparirà allora strano che per comprendere il tema del Concilio e la contestazione si sia ricorso a diverse profondità, dalla più vicina (il con-

${ }^{45}$ D. Saresella, Dal Concilio alla contestazione. Riviste cattoliche negli anni del cambiamento (1958-1968), Morcelliana, Brescia 2005, p. 461 e nota 423.

${ }^{46}$ D. Saresella, Cattolici a sinistra. Dal modernismo ai nostri giorni, Laterza, RomaBari 2011, pp. 141-142.

${ }^{47}$ M. Ranchetti, Scritti diversi, IV. Ulteriori e ultimi (2000-2008), Edizioni di Storia e Letteratura, Roma 2010, pp. 159-207.

${ }^{48}$ M. Pacioni, Ranchetti, Michele, in Dizionario biografico degli italiani, vol. 86, Istituto della Enciclopedia italiana, Roma 2016, p. 375. 
N.B: Copia ad uso personale. È vietata la riproduzione (totale o parziale) dell'opera con qualsiasi mezzo effettuata e la sua messa a disposizione di terzi, sia in forma gratuita sia a pagamento.

cilio stesso) a quella forse oggi meno evidente (il modernismo), fino a studi che sono anche testimonianze, nelle quali si riuniscono tre dei problemi emersi finora: il rapporto tra chierici, ex-chierici e laici, l'assoluta centralità del nesso tra biografia, storiografia e autobiografia e l'uso di contestazione, dissenso e protesta. Non conosco un esempio migliore dell'intreccio di questi temi di quello fornito da Giuseppe Gozzini nel suo libro Sulla frontiera. Camillo De Piaz, la Resistenza, il Concilio e oltre, stampato nel 2006, che analizzerò rapidamente, affiancandolo all'Autobiografia di un cattolico marginale di Giovanni Franzoni e muovendo dal modo con cui il volume fu allestito:

da una decina d'anni, per gravi disturbi alla vista, padre Camillo non riesce più a leggere né a scrivere, una dura condanna per un uomo di attente e pensose letture, per un sacerdote abituato a scrivere anche le sue omelie. Gli è rimasta la conversazione, intercalata dai suoi proverbiali silenzi. Nella sofferta "solitudine istituzionale", il suo pensiero si è ancor più affinato. Sulle passate esperienze abbiamo ritrovato una consonanza nella vecchiaia - incipiente per me, avanzata per lui - e la ricostruzione della memoria approdava a momenti di opaca, ma non perduta lontananza, alternati a bagliori di lucida speranza. È in atto una cancellazione organizzata della memoria non solo per decostruire e adattare al presente il patrimonio della Resistenza antifascista, ma anche per cancellare e rendere innocua la cultura cattolica degli anni cinquanta, che ha preannunciato e preparato il Concilio. Contro questa rimozione forzata della memoria ha più di qualcosa da dire padre Camillo, con l'irriducibilità della sua storia personale, all'ammucchiata indistinta della retorica della resistenza e alle frettolose interpretazioni del dissenso cattolico ${ }^{49}$.

Come l'Autobiografia di Franzoni («trascritta da dettatura registrata perché il protagonista ha ormai perso quasi totalmente l'uso della vista», ma non della «voce profetica») $)^{50}$, Sulla frontiera si presenta come un processo verbale legato al declino di due testimoni. Ma, almeno in un caso, colui che dice "io" insieme al testimone è esso stesso un testimone degli anni del Concilio e della "contestazione". Nel novembre del 1962, Giovanni Gozzini fu il primo obiettore cattolico italiano. Incarcerato e processato, fu difeso da Ernesto Balducci, Lorenzo Milani, David Maria Turoldo e Camillo De Piaz. Messo a confronto con De Piaz (nato a Tirano nel 1918 e mancato nel 2010, animatore, insieme a padre David, della Corsia dei Servi

${ }^{49}$ G. Gozzini, Sulla frontiera: Camillo De Piaz, la Resistenza, il Concilio e oltre, Libri Scheiwiller, Milano 2006, p. 11.

${ }^{50}$ Franzoni, Autobiografia, cit., p. 13. 
N.B: Copia ad uso personale. È vietata la riproduzione (totale o parziale) dell'opera con qualsiasi mezzo effettuata e la sua messa a disposizione di terzi, sia in forma gratuita sia a pagamento.

a Milano, fino al suo allontanamento nel 1957$)^{51}$, Gozzini intreccia un dialogo con quello che è l'oggetto del volume che forma un flusso continuo, con una sola, vistosa eccezione, che andrebbe riportata per intero, ma di cui è possibile isolare un passaggio tra i più significativi, dove la "storia" prende il posto del passato:

torniamo alla loro storia. È insieme agli Ordini di cui si è parlato che gli ultimi tra i Fondatori, con San Filippo Benizi, devono affrontare la battaglia per sopravvivere, dopo che, nel rigurgito della vecchia mentalità e delle vecchie strutture, del vecchio ordine e del vecchio costume minacciati, un decreto del Concilio di Lione (1274) rimette in discussione la legittimità delle nuove forme religiose, che si erano chiamate, polemicamente, mendicanti, quasi ad accentuare anche nel nome l'esigenza, di cui erano portatrici e promotrici, di una liberazione della Chiesa dalle sue compromissioni temporali e politiche, da una sua identificazione con una struttura, quella feudale, fondata essenzialmente sulla proprietà fondiaria, la spinta verso un ritorno alle origini, al "puro Vangelo"s2.

La citazione è tratta da un'omelia sull'origine dei Servi di Maria nel XIII secolo, pronunciata nel 1980 e pubblicata nel $1983^{53}$. Molto lontana dall'accertamento documentario condotto dagli stessi membri dell'Ordine a proposito delle origini dei Servi ${ }^{54}$, essa spiega infine come era possibile intendere la "contestazione": il Vaticano II come cesura irreversibile ed il passato anche più remoto del cristianesimo e del cattolicesimo romano come una miniera nella quale scoprire un nuovo inizio.

Fino a che punto sia possibile risalire agli inizi, lo mostra, peraltro, uno degli ultimi libri apparsi in traduzione italiana di Leonardo Boff ${ }^{55}$. Abbandonato l'ordine dei frati Minori nel 1992, Boff ha continuato a «sentirsi francescano ${ }^{56}$, ma in maniera molto diversa da come Camillo De Piaz si sentiva servo di Maria. Entrato quasi per caso (stando al suo racconto)

${ }^{51}$ Per la prima parte della biografia di De Piaz e l'esperienza della Corsia si veda D. Saresella, David M. Turoldo, Camillo De Piaz e la Corsia dei Servi di Milano (19431963), Morcelliana, Brescia 2008.

${ }^{52}$ Gozzini, Sulla frontiera, cit., pp. 26-27.

${ }^{53}$ C. De Piaz, Omelia per la festa dei primi sette padri, in «Servitium», 1983, 26-27, pp. 55-61.

${ }^{54}$ Come mostrano le voci esemplari di Franco Dal Pino: Filippo Benizi, santo, in Dizionario biografico degli italiani, vol. 47, Istituto della Enciclopedia italiana, Roma 1997, pp. 723-727, e Manetto da Firenze, santo, ivi, vol. 68, Istituto della Enciclopedia italiana, Roma 2007, pp. 629-631.

${ }^{55}$ L. Boff, L. Zoja, Tra eresia e verità, Chiarelettere, Milano 2014.

${ }^{56}$ Ivi, p. 69. 
N.B: Copia ad uso personale. È vietata la riproduzione (totale o parziale) dell'opera con qualsiasi mezzo effettuata e la sua messa a disposizione di terzi, sia in forma gratuita sia a pagamento.

tra $\mathrm{i}$ frati Minori in Brasile, formatosi a Monaco di Baviera, inizialmente con Karl Rahner, in rapporti cordiali con il giovane Joseph Ratzinger ${ }^{57}$, l'ultimo Boff considera il francescanesimo «una dimensione dello spirito» e Francesco «un archetipo dell'Occidente e forse di tutto il mondo» ${ }^{58}$. Qui e altrove nel libro la parola archetipo è usata in senso tecnico, né potrebbe essere diversamente, trattandosi di un dialogo con lo psicanalista junghiano Luigi Zoja.

Quale posto occupa in tutto ciò la storia (intesa come ricerca critica condotta dal presente, volgendosi verso il passato)? Nessuno. In questa nuova geografia, tra Italia e America latina, il Concilio Vaticano II e la "contestazione" sono collocati sullo sfondo e diventano una memoria di un paese lontano, in cui un uomo come Francesco, vissuto nel XII-XIII secolo diventa uno sfondo. O meglio, un archetipo.

${ }^{57}$ Ivi, pp. 31-40.

${ }^{58}$ Ivi, p. 87. 